\title{
SIGNAL RECONSTRUCTION FROM SINE WAVE CROSSINGS
}

\author{
Holger Boche and Ullrich J. Mönich \\ Technische Universität München \\ Lehrstuhl für Theoretische Informationstechnik \\ 80290 München, Germany
}

\begin{abstract}
In this paper we analyze the reconstruction of bandlimited signals from their sine wave crossings by a sampling type reconstruction process. The reconstruction process is highly adapted to the signal which shall be reconstructed, because the reconstruction functions and the sampling points are implicitly generated by the signal. We show that the reconstruction process is uniformly convergent for all signals in the Paley-Wiener spaces $\mathcal{P} \mathcal{W}_{\pi}^{p}, 1<p \leq \infty$. However, the adaptivity cannot prevent the peak value of the reconstruction process to diverge for certain signals in $\mathcal{P} \mathcal{W}_{\pi}^{1}$.
\end{abstract}

Index Terms - adaptive, reconstruction, sampling, sine type, zero crossings

\section{INTRODUCTION}

The recovery of bandlimited signals from their zero crossing has been intensively studied, because the zero crossings of a signal are robust under various non-linear disturbances the signal might undergo, for example when it is transmitted over a non-linear channel or in magnetic recording [1]. According to Hadamard's factorization theorem [2, p.26], a bandlimited signal that is bounded on the real axis is - up to some constants - uniquely determined by its zeros [3]. In general the zeros of a bandlimited signal are complex, but in physical systems, complex zeros are not observable [4]. Only the zero crossings, i.e., the locations of the odd order real zeros of a signal, can be easily detected. But, in general, the zero crossings do not contain enough information to reconstruct the signal [5]. As the example $(1-\operatorname{sinc}(\pi t)) / t^{2}$ shows, there are even bandlimited signals that have no real zero at all. In order to circumvent the problem of complex and multiple zeros, the invertible transformation

$$
\psi_{f}(z)=f(z)-A \sin (\pi z), \quad z \in \mathbb{C},
$$

with $A>\|f\|_{\infty}$, has been considered [5, 6, 7]. For signals $f$ that are bandlimited with bandwidth not larger than $\pi$, and both bounded and real on the real axis, $\psi_{f}$ has only real and simple zeros [8]. Moreover, given such a signal $f$, the theory of entire functions shows that $\psi_{f}$ can be reconstructed from its real zero crossings $\left\{t_{k}\right\}_{k \in \mathbb{Z}}$ by virtue of the infinite product

$$
\psi_{f}(z)=\psi_{f}(0) \lim _{R \rightarrow \infty} \prod_{\left|t_{k}\right| \leq R}\left(1-\frac{z}{t_{k}}\right)
$$

under the assumption that $\psi_{f}(0) \neq 0$. Thus, $\psi_{f}$ is determined by its zeros $\left\{t_{k}\right\}_{k \in \mathbb{Z}}$ and $\psi_{f}(0)=f(0)$. Since $\psi_{f}\left(t_{k}\right)=f\left(t_{k}\right)-$ $A \sin \left(\pi t_{k}\right)=0, k \in \mathbb{Z}$, the zero crossings $\left\{t_{k}\right\}_{k \in \mathbb{Z}}$ of $\psi_{f}$ are the sine wave crossings of $f$. Finally, the signal $f$ can be reconstructed

This work was supported by the German Research Foundation (DFG) under grant BO 1734/9-1. We also would like to thank the TU-München for providing start-up funds. from $\psi_{f}$ by using (1). Hence, $f$ is uniquely determined by its sine wave crossings $\left\{t_{k}\right\}_{k \in \mathbb{Z}}$ and $f(0)$.

There is a considerable amount of literature dealing with the reconstruction of signals from its zeros by products $[9,3,7]$. In general the convergence of infinite products is intricate and needs a careful treatment. However, in many publications the argumentation is more or less heuristic, and the results are rather claims without a rigorous mathematical justification. Although, under the assumptions on the signal $f$ from above, the function $\psi_{f}$ and consequently the signal $f$ are uniquely determined by $\left\{t_{k}\right\}_{k \in \mathbb{Z}}$ and $f(0)$, the question remains whether the reconstruction is stable.

One of the first papers that presents a mathematically rigorous approach is [6]. In [6], Bar-David showed that every bandlimited signal $f$ with bandwidth smaller than $\pi$ that is bounded and real on the real axis, and that satisfies $f(0) \neq 0$, can be reconstructed according to (1) and (2) from the sine wave crossings of $f$, i.e., the real and simple zeros of $\psi_{f}$, and $f(0)$, provided that $A>\|f\|_{\infty}$.

In practice the reconstruction of $\psi_{f}$ using the infinite product according to (2) has several drawbacks in terms of convergence speed an sensitivity against jitter in the location of the zeros $\left\{t_{k}\right\}_{k \in \mathbb{Z}}$, and thus is not the preferred way to do the reconstruction.

\section{NOTATION}

Let $\hat{f}$ denote the Fourier transform of a function $f$, where $\hat{f}$ is to be understood in the distributional sense. $L^{p}(\mathbb{R}), 1 \leq p<\infty$, is the space of all $p$ th-power Lebesgue integrable functions on $\mathbb{R}$, with the usual norm $\|\cdot\|_{p}$, and $L^{\infty}(\mathbb{R})$ is the space of all functions for which the essential supremum norm $\|\cdot\|_{\infty}$ is finite.

For $\sigma>0$ let $\mathcal{B}_{\sigma}$ be the set of all entire functions $f$ with the property that for all $\epsilon>0$ there exists a constant $C(\epsilon)$ with $|f(z)| \leq$ $C(\epsilon) \exp ((\sigma+\epsilon)|z|)$ for all $z \in \mathbb{C}$. The Bernstein space $\mathcal{B}_{\sigma}^{p}$ consists of all functions in $\mathcal{B}_{\sigma}$, whose restriction to the real line is in $L^{p}(\mathbb{R})$, $1 \leq p \leq \infty$ [10]. The norm for $\mathcal{B}_{\sigma}^{p}$ is given by the $L^{p}$-norm on the real line, i.e., $\|\cdot\|_{\mathcal{B}_{\sigma}^{p}}=\|\cdot\|_{p}$. A signal in $\mathcal{B}_{\sigma}^{p}$ is called bandlimited to $\sigma$. By the Paley-Wiener-Schwartz theorem the Fourier transform of a signal bandlimited to $\sigma$ is supported in $[-\sigma, \sigma][10]$. For $1 \leq$ $p \leq 2$ the Fourier transformation is defined in the classical and for $p>2$ in the distributional sense.

For $\sigma>0$ and $1 \leq p \leq \infty$ we denote by $\mathcal{P} \mathcal{W}_{\sigma}^{p}$ the Paley-Wiener space of signals $f$ with a representation $f(z)=$ $1 /(2 \pi) \int_{-\sigma}^{\sigma} g(\omega) \mathrm{e}^{i z \omega} \mathrm{d} \omega, z \in \mathbb{C}$, for some $g \in L^{p}[-\sigma, \sigma]$. If $f \in \mathcal{P} \mathcal{W}_{\sigma}^{p}$ then $g(\omega)=\hat{f}(\omega)$. The norm for $\mathcal{P} \mathcal{W}_{\sigma}^{p}, 1 \leq p<\infty$, is given by $\|f\|_{\mathcal{P} \mathcal{W}_{\sigma}^{p}}=\left(1 /(2 \pi) \int_{-\sigma}^{\sigma}|\hat{f}(\omega)|^{p} \mathrm{~d} \omega\right)^{1 / p}$. For $p=2$ we obtain the Paley-Wiener space $\mathcal{P} \mathcal{W}_{\sigma}^{2}$, which is nothing else than the space of bandlimited signals with finite energy. Hölder's inequality leads to $\mathcal{P} \mathcal{W}_{\sigma}^{s} \subset \mathcal{P} \mathcal{W}_{\sigma}^{p}$ for $1 \leq p<s \leq \infty$. Moreover, we have $\|f\|_{\infty} \leq\|f\|_{\mathcal{P} \mathcal{W}_{\sigma}^{1}}$, which implies that every signal in $\mathcal{P} \mathcal{W}_{\sigma}^{1}$ is bounded on the real line. 


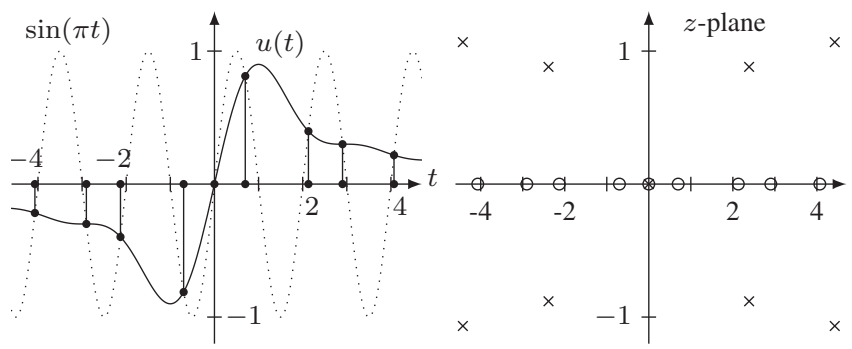

(a) Plot of $u(t)$ (solid line) and $\sin (\pi t)$ (b) Plot of the zeros of $u(z)(\times)$ (dotted line). and the zeros of $\psi_{u}(z)(0)$ in the
complex plane.

Fig. 1. Illustration of the transformation of the complex zeros of a bandlimited signal in real zeros.

\section{CONVERGENCE RESULT}

\subsection{Basic Properties of $\psi_{f}$}

In the introduction we have seen that the transformation (1) is important, because for signals $f \in \mathcal{B}_{\pi}^{\infty}$ that are real on the real axis, $\psi_{f}$ has only real and simple zeros. Figure 1 illustrates this transformation. The signal $u(t)=(1-\operatorname{sinc}(\pi t)) / t$, which is depicted in Fig. 1(a), has only complex zeros except for one real zero at zero. The zeros of $u$ in the complex plane in the vicinity of the origin are plotted in Fig. 1(b) as crosses. Furthermore, the zeros of $\psi_{f}$, which are plotted as circles in Fig. 1(b), are nothing else than the abscissas of the crossings of $u$ and the sine function.

In the following we use the transformation (1) only for signals $f \in \mathcal{P} \mathcal{W}_{\pi}^{1}$ that are real on the real axis, and we assume that $A=1$, i.e., we consider the transformation

$$
\psi_{f}(z)=f(z)-\sin (\pi z) .
$$

The space $\mathcal{P} \mathcal{W}_{\pi}^{1}$ is interesting, because it is the largest space in the scale of Paley-Wiener spaces.

For signals $f \in \mathcal{P} \mathcal{W}_{\pi}^{1}$ that are real on the real axis and that satisfy $\|f\|_{\infty}<1, \psi_{f}$ is a sine-type function. This fact will be of value for us, because sine-type functions have many pleasant properties.

Definition 1. An entire function $f$ of exponential type $\pi$ is said to be of sine type if

(i) the zeros of $f$ are separated and simple, and

(ii) there exist positive constants $A, B$, and $H$ such that $A \mathrm{e}^{\pi|y|} \leq$ $|f(x+i y)| \leq B \mathrm{e}^{\pi|y|}$ whenever $x$ and $y$ are real and $|y| \geq \bar{H}$.

Example 1. $\sin (\pi z)$ is a function of sine type and its zeros are $t_{k}=$ $k, k \in \mathbb{Z}$.

In the following discussion we will show that $\psi_{f}$ is a sine-type function if $f$ is in $\mathcal{P} \mathcal{W}_{\pi}^{1}$, satisfies $\|f\|_{\infty}<1$, and is real on the real axis. From [8] we know that $\psi_{f}$ has only simple zeros. Moreover, since $\lim _{|t| \rightarrow \infty} f(t)=0$ according to the Riemann-Lebesgue lemma, it follows that the zeros are also separated. Thus, $\psi_{f}$ satisfies item (i) of Definition 1. Moreover, since $\psi_{f}$ has the representation $\psi_{f}(t)=\frac{1}{2 \pi} \int_{-\pi}^{\pi} \mathrm{e}^{i \omega t} \mathrm{~d} \mu(\omega)$, where $\mu(\omega)$ is of bounded variation on $[-\pi, \pi]$ and has a jump discontinuity at each endpoint, it follows $\left[11\right.$, p. 143] that $\psi_{f}$ also satisfies item (ii) of Definition 1, and hence is a sine-type function.

For further information about sine-type functions we would like to refer the reader to [11] and [2].

\subsection{Adaptive Reconstruction Process}

In the previous section we have seen that bandlimited signals $f \in$ $\mathcal{P} \mathcal{W}_{\pi}^{1},\|f\|_{\mathcal{P} \mathcal{W}_{\pi}^{1}}<1$, that are real on the real axis can be reconstructed from their sine wave crossings, i.e., the zero crossings of $\psi_{f}$, by using (2) and (3) as reconstruction formulas. In this paper we analyze a different reconstruction process, which has the shape

$$
\sum_{k=-\infty}^{\infty} f\left(\lambda_{k}\right) \frac{\psi_{f}(t)}{\psi_{f}^{\prime}\left(\lambda_{k}\right)\left(t-\lambda_{k}\right)}
$$

where $\psi_{f}$ is defined as in (3), and $\left\{\lambda_{k}\right\}_{k \in \mathbb{Z}}$ denotes the zero sequence of $\psi_{f}$. Note that the knowledge of the zero sequence of $\psi_{f}$ is enough for the reconstruction process (4). The samples $\left\{f\left(\lambda_{k}\right)\right\}_{k \in \mathbb{Z}}$ can be calculated from $\psi_{f}$ : since $\psi_{f}\left(\lambda_{k}\right)=0, k \in \mathbb{Z}$, it follows from (3) that $f\left(\lambda_{k}\right)=\sin \left(\lambda_{k}\right), k \in \mathbb{Z}$.

Reconstruction processes of the shape (4) are a special case of more general sampling series with non-equidistant sampling patterns of the shape $\sum_{k=-\infty}^{\infty} f\left(\lambda_{k}\right) \phi_{k}(t)$, where $\phi_{k}, k \in \mathbb{Z}$, are certain reconstruction functions, and $\left\{\lambda_{k}\right\}_{k \in \mathbb{Z}}$ is the sequence of sampling points [12]. In our case, the sampling pattern $\left\{\lambda_{k}\right\}_{k \in \mathbb{Z}}$ and the reconstruction functions

$$
\phi_{f, k}(t):=\frac{\psi_{f}(t)}{\psi_{f}^{\prime}\left(\lambda_{k}\right)\left(t-\lambda_{k}\right)}
$$

are implicitly generated by the signal $f$, and thus, in a certain sense, adapted to the signal $f$.

For a large class of signals the reconstruction process (4) converges uniformly on all of $\mathbb{R}$.

Theorem 1. Let $1<p \leq \infty$. For all $f \in \mathcal{P} \mathcal{W}_{\pi}^{p}$ for which $\psi_{f}$ is a sine-type function we have

$$
\lim _{N \rightarrow \infty}\left(\sup _{t \in \mathbb{R}}\left|f(t)-\sum_{k=-N}^{N} f\left(\lambda_{k}\right) \frac{\psi_{f}(t)}{\psi_{f}^{\prime}\left(\lambda_{k}\right)\left(t-\lambda_{k}\right)}\right|\right)=0,
$$

where $\left\{\lambda_{k}\right\}_{k \in \mathbb{Z}}$ denotes the zero sequence of $\psi_{f}$.

The proof of Theorem 1 is based on the following lemma, a proof of which can be found in [2, p. 165].

Lemma 1. Let $\phi$ be a function of sine type, and $\left\{\lambda_{k}\right\}_{k \in \mathbb{Z}}$ its zero set. Further, let $1<p<\infty$. Then there exist two constants $C_{L}(p)>0$ and $C_{R}(p)>0$, depending only on $p$, such that for all $f \in \mathcal{B}_{\pi}^{p}$

$$
C_{L}(p)\left(\sum_{k=-\infty}^{\infty}\left|f\left(\lambda_{k}\right)\right|^{p}\right)^{\frac{1}{p}} \leq\|f\|_{\mathcal{B}_{\pi}^{p}} \leq C_{R}(p)\left(\sum_{k=-\infty}^{\infty}\left|f\left(\lambda_{k}\right)\right|^{p}\right)^{\frac{1}{p}} .
$$

Proof of Theorem 1. Since $\mathcal{P} \mathcal{W}_{\pi}^{p} \subset \mathcal{P} \mathcal{W}_{\pi}^{2}$ for $p \geq 2$, it is clear that if (5) is true for all $f \in \mathcal{P} \mathcal{W}_{\pi}^{2}$ for which $\psi_{f}$ is a sine-type function, then (5) is true for all $f \in \mathcal{P} \mathcal{W}_{\pi}^{p}, p \geq 2$, for which $\psi_{f}$ is a sine-type function. Hence, it suffices to prove the case $1<p \leq 2$.

Let $1<p \leq 2$. Further, let $f \in \mathcal{P} \mathcal{W}_{\pi}^{p}$ be such that $\psi_{f}$ is a sine-type function. Since $f \in \mathcal{B}_{\pi}^{q}$ and $\phi_{f, k} \in \mathcal{B}_{\pi}^{q}, 1 / p+1 / q=1$, we can apply Lemma 1 to obtain

$$
\begin{aligned}
\| f & -\sum_{k=-N}^{N} f\left(\lambda_{k}\right) \phi_{f, k} \|_{\mathcal{B}_{\pi}^{q}} \\
& \leq C_{R}(q)\left(\sum_{l=-\infty}^{\infty}\left|f\left(\lambda_{l}\right)-\sum_{k=-N}^{N} f\left(\lambda_{k}\right) \phi_{f, k}\left(\lambda_{l}\right)\right|^{q}\right)^{\frac{1}{q}} \\
& \leq C_{R}(q)\left(\sum_{|l|>N}\left|f\left(\lambda_{l}\right)\right|^{q}\right)^{\frac{1}{q}}
\end{aligned}
$$


for all $N \in \mathbb{N}$. In the last inequality we used the interpolation property of $\phi_{f, k}$, namely $\phi_{f, k}\left(\lambda_{l}\right)=1$ if $l=k$, and $\phi_{f, k}\left(\lambda_{l}\right)=0$ if $l \neq k$. Moreover, since

$$
\left(\sum_{l=-\infty}^{\infty}\left|f\left(\lambda_{l}\right)\right|^{q}\right)^{\frac{1}{q}} \leq \frac{\|f\|_{\mathcal{B}_{\pi}^{q}}}{C_{L}(q)}<\infty
$$

it follows that

$$
\lim _{N \rightarrow \infty}\left(\sum_{|l|>N}\left|f\left(\lambda_{l}\right)\right|^{q}\right)^{\frac{1}{q}}=0
$$

The proof is complete, because there exists a constant $C(q)$ such that $\|f\|_{\infty} \leq C(q)\|f\|_{\mathcal{B}_{\pi}^{q}}$ for all $f \in \mathcal{B}_{\pi}^{q}$.

Remark 1. In Theorem 1 we have the requirement that $\psi_{f}$ is a sinetype function. A simple sufficient condition for $\psi_{f}$ to be a sine-type function is that the signal $f \in \mathcal{P} \mathcal{W}_{\pi}^{p}, 1<p \leq \infty$, is real on the real axis and $\|f\|_{\infty}<1$.

Remark 2. For fixed $t \in \mathbb{R}$, the sum in Theorem 1 is absolutely and unconditionally convergent.

\section{DIVERGENCE RESULT}

In [13] we conjectured that the sampling series with matched reconstruction function (4) is not uniformly convergent on all of $\mathbb{R}$ for certain $f \in \mathcal{P} \mathcal{W}_{\pi}^{1}$ with $\|f\|_{\mathcal{P} \mathcal{W}_{\pi}^{1}}<1$. Next, we will prove that this conjecture is true. This shows that the restriction in Theorem 1 that $f \in \mathcal{P} \mathcal{W}_{\pi}^{p}$ with $p$ strictly larger than 1 is indeed necessary. Thus, Theorem 1 is sharp in the sense that it cannot be extended to hold for $p=1$.

Theorem 2. There exists a function $f_{1} \in \mathcal{P} \mathcal{W}_{\pi}^{1}$, such that $\psi_{f_{1}}$ is a sine-type function with solely real zeros and

$$
\limsup _{N \rightarrow \infty}\left(\sup _{t \in \mathbb{R}}\left|f_{1}(t)-\sum_{k=-N}^{N} f_{1}\left(t_{k}\right) \frac{\psi_{f_{1}}(t)}{\psi_{f_{1}}^{\prime}\left(t_{k}\right)\left(t-t_{k}\right)}\right|\right)=\infty,
$$

where $\left\{t_{k}\right\}_{k \in \mathbb{Z}} \subset \mathbb{R}$ denotes the zero sequence of $\psi_{f_{1}}$.

Theorem 2 shows that even the adaptivity of the reconstruction process (4) to the signals cannot avoid the divergence of the peak value of reconstruction error for certain signals in $\mathcal{P} \mathcal{W}_{\pi}^{1}$.

For the proof of Theorem 2 we use a recent result by Hryniv and Mykytyuk, which was proved in [14].

Theorem 3 (Hryniv and Mykytyuk).

(i) Let $w \in \mathcal{P} \mathcal{W}_{\pi}^{1}$ and $\lambda_{k}=k+w(k), k \in \mathbb{Z}$. Then there exists a function $f \in \mathcal{P} \mathcal{W}_{\pi}^{1}$ such that $\psi_{f}$ has the zero sequence $\left\{\lambda_{k}\right\}_{k \in \mathbb{Z}}$

(ii) Conversely, if $f \in \mathcal{P} \mathcal{W}_{\pi}^{1}$, then there exists a function $\omega \in$ $\mathcal{P} \mathcal{W}_{\pi}^{1}$ such that $\left\{\lambda_{k}\right\}_{k \in \mathbb{Z}}=\{k+w(k)\}_{k \in \mathbb{Z}}$ is the zeros sequence of $\psi_{f}$.

This theorem is remarkable, because it completely characterizes the zero sequences of $\psi_{f}$ for $f \in \mathcal{P} \mathcal{W}_{\pi}^{1}$ in terms of a disturbance of the integers by a function in $\mathcal{P} \mathcal{W}_{\pi}^{1}$. In general it is hard to find such descriptions for the zeros of entire functions.

The following corollary is a simple consequence of Theorem 3 .

Corollary 1. Let $w \in \mathcal{P} \mathcal{W}_{\pi}^{1}$ be real on the real axis with $|w(k)|<$ $1 / 2, k \in \mathbb{Z}$, and $t_{k}=k+w(k), k \in \mathbb{Z}$. Then there exists a function $f \in \mathcal{P} \mathcal{W}_{\pi}^{1}$ that is real on the real axis such that $\psi_{f}$ is a function of sine type that has the zero sequence $\left\{t_{k}\right\}_{k \in \mathbb{Z}}$.
Proof. Let $w \in \mathcal{P} \mathcal{W}_{\pi}^{1}$ be real on the real axis with $|w(k)|<1 / 2$, $k \in \mathbb{Z}$, and $t_{k}=k+w(k), k \in \mathbb{Z}$. According to part (i) of Theorem 3 , there exists a function $f \in \mathcal{P} \mathcal{W}_{\pi}^{1}$ such that $\psi_{f}$ has the zero sequence $\left\{t_{k}\right\}_{k \in \mathbb{Z}}$. Since $\left\{t_{k}\right\}_{k \in \mathbb{Z}} \subset \mathbb{R}$, it follows from (2) that $\psi_{f}$ and consequently $f$ can be chosen to be real on the real axis. It remains to show that $\psi_{f}$ is a sine-type function. Since $|w(k)|<1 / 2$, $k \in \mathbb{Z}$, and $\lim _{|k| \rightarrow \infty} w(k)=0$, the zeros $\left\{t_{k}\right\}_{k \in \mathbb{Z}}$ of $\psi_{f}$ are separated and simple, hence $\psi_{f}$ satisfies item (i) of Definition 1. Moreover, by the same argumentations as in Sec. 3.1, $\psi_{f}$ also satisfies item (ii) of Definition 1, and hence is a sine-type function.

For the proof of Theorem 2, we need the following lemmas.

Lemma 2. Let $f \in \mathcal{P} \mathcal{W}_{\pi}^{1}$ be such that $\psi_{f}$ is a sine-type function. Then there exists a constant $C_{1}$ such that for all $N \in \mathbb{N}$ sufficiently large we have $\left|\psi_{f}\left(N+\frac{1}{2}\right)\right| \geq C_{1}$.

Proof. Let $\left\{\lambda_{k}\right\}_{k \in \mathbb{Z}}$ be the zero sequence of $\psi_{f}$. Since $\psi_{f}$ is a sinetype function, there exists, for every $\epsilon>0$, a number $C_{2}>0$ such that $\left|\psi_{f}(x+i y)\right| \geq C_{2} \mathrm{e}^{\pi|y|}$ outside the circles of radius $\epsilon$ centered at the zeros $\left\{\lambda_{k}\right\}_{k \in \mathbb{Z}}$ of $\psi_{f}$ [11, p. 144, Lemma 2]. According to part (ii) of Theorem 3 we have $\lambda_{k}=k+w(k), k \in \mathbb{Z}$, for some $w \in \mathcal{P} \mathcal{W}_{\pi}^{1}$. Further, since $\lim _{k \rightarrow \infty} w(k)=0$, there exists a natural number $N_{0}$ such that $\left|N+1 / 2-\lambda_{N}\right| \geq 1 / 4$ for all $N \geq N_{0}$.

Lemma 3. Let $f \in \mathcal{P} \mathcal{W}_{\pi}^{1}$, and let $\left\{\lambda_{k}\right\}_{k \in \mathbb{Z}}$ denote the zero sequence of $\psi_{f}$. Then for all sequences $\left\{a_{k}\right\}_{k \in \mathbb{Z}} \subset \mathbb{C}$ with $\sup _{k \in \mathbb{Z}}\left|a_{k}\right|<\infty$ there exists a constant $C_{3}$ such that for all sufficiently large $N$ we have

$$
\left|\sum_{k=-N}^{N} \frac{a_{k}}{\left(N+\frac{1}{2}-\lambda_{k}\right)}-\sum_{k=-N}^{N} \frac{a_{k}}{\left(N+\frac{1}{2}-k\right)}\right| \leq C_{3} .
$$

Proof. According to part (ii) of Theorem 3 there exists a function $w \in \mathcal{P} \mathcal{W}_{\pi}^{1}$ such that $\lambda_{k}=k+w(k), k \in \mathbb{Z}$. For sufficiently large $N \in \mathbb{N}$ we have $N+1 / 2-\lambda_{k} \geq 1 / 4$ for all $k \in \mathbb{Z}$. Further, since $w \in \mathcal{P} \mathcal{W}_{\pi}^{1}$, there exists a $N_{0} \in \mathbb{N}$ such that $|w(k)|<1 / 4$ for all $k \geq N_{0}$. Thus, for sufficiently large $N, N \geq N_{0}$, we have

$$
\begin{aligned}
& \left|\sum_{k=-N}^{N} \frac{a_{k}}{N+\frac{1}{2}-\lambda_{k}}-\sum_{k=-N}^{N} \frac{a_{k}}{N+\frac{1}{2}-k}\right| \\
& \leq\|w\|_{\mathcal{P} \mathcal{W}_{\pi}^{1}} \sup _{k \in \mathbb{Z}}\left|a_{k}\right| \sum_{k=-N}^{N} \frac{1}{\left(N+\frac{1}{2}-\lambda_{k}\right)\left(N+\frac{1}{2}-k\right)} .
\end{aligned}
$$

Finally, using elementary calculations it can be shown that the sum in (6) is bounded from above by some constant.

Lemma 4. There exists a function $w \in \mathcal{P} \mathcal{W}_{\pi}^{1}$ that is real on the real axis with $\|w\|_{\mathcal{P} \mathcal{W}_{\pi}^{1}}<1 / 2$ and $w(k) \geq 0, k \in \mathbb{Z}$, such that

$$
\limsup _{N \rightarrow \infty} \sum_{k=-N}^{N} \frac{\sin (\pi w(k))}{N+\frac{1}{2}-k}=\infty \text {. }
$$

Proof. For $N \in \mathbb{N}$, consider the function

$$
f_{N}(t):=\sum_{k=-2 N+1}^{2 N-1} f_{N}(k) \frac{\sin (\pi(t-k))}{\pi(t-k)}
$$

where $f_{N}(k)=1$ for $|k| \leq N$, and $f_{N}(k)=2-|k| / N$ for $N<$ $|k|<2 N$. A short calculation shows that $\left\|f_{N}\right\|_{\mathcal{P} \mathcal{W}_{\pi}^{1}} \leq 3, N \in \mathbb{N}$. Next, let $M(l)=2^{\left(l^{3}\right)}, l \in \mathbb{Z}$, and choose

$$
w(t)=\frac{1}{10} \sum_{l=1}^{\infty} \frac{1}{l^{2}} f_{M(l)}(t) .
$$


It follows that $w \in \mathcal{P} \mathcal{W}_{\pi}^{1}$, because

$$
\|w\|_{\mathcal{P} \mathcal{W}_{\pi}^{1}} \leq \frac{1}{10} \sum_{l=1}^{\infty} \frac{1}{l^{2}}\left\|f_{M(l)}\right\|_{\mathcal{P} \mathcal{W}_{\pi}^{1}} \leq \frac{3}{10} \frac{\pi^{2}}{6}<\frac{1}{2},
$$

and that $w(k) \geq 0, k \in \mathbb{Z}$. Consequently, we have $0 \leq w(k)<$ $1 / 2$, for all $k \in \mathbb{Z}$. Thus, for $r \in \mathbb{N}$, we have

$$
\begin{aligned}
& \sum_{k=-M(r)}^{M(r)} \frac{\sin (\pi w(k))}{M(r)+\frac{1}{2}-k} \geq 2 \sum_{k=-M(r)}^{M(r)} \frac{w(k)}{M(r)+\frac{1}{2}-k} \\
& \geq \frac{1}{5 r^{2}} \sum_{k=-M(r)}^{M(r)} \frac{f_{M(r)}(k)}{M(r)+\frac{1}{2}-k}=\frac{1}{5 r^{2}} \sum_{k=0}^{2 M(r)} \frac{1}{k+\frac{1}{2}} \\
& >\frac{1}{5 r^{2}} \log (M(r))=\frac{1}{5} r \log (2),
\end{aligned}
$$

because $\sin (x) \geq 2 x / \pi$ for all $x \in[0, \pi / 2]$.

Now, we are in the position to prove Theorem 2.

Proof of Theorem 2. Let $w \in \mathcal{P} \mathcal{W}_{\pi}^{1}$ be the function from Lemma 4 and $\left\{t_{k}\right\}_{k \in \mathbb{Z}}=\{k+w(k)\}_{k \in \mathbb{Z}} \subset \mathbb{R}$. According to Corollary 1 there exists a function $f_{1} \in \mathcal{P} \mathcal{W}_{\pi}^{1}$ that is real on the real axis such that $\psi_{f_{1}}$ is a function of sine type that has the zero sequence $\left\{t_{k}\right\}_{k \in \mathbb{Z}}$. Therefore, we have $f_{1}\left(t_{k}\right)=\sin \left(\pi t_{k}\right)=(-1)^{k} \sin (\pi w(k))$ for all $k \in \mathbb{Z}$. For $N \in \mathbb{N}$, sufficiently large, so that Lemma 2 can be applied, it follows that

$$
\begin{aligned}
& \left|\sum_{k=-N}^{N} f_{1}\left(t_{k}\right) \frac{\psi_{f_{1}}\left(N+\frac{1}{2}\right)}{\psi_{f_{1}}^{\prime}\left(t_{k}\right)\left(N+\frac{1}{2}-t_{k}\right)}\right| \\
& \quad=\left|\psi_{f_{1}}\left(N+\frac{1}{2}\right)\right|\left|\sum_{k=-N}^{N} \frac{(-1)^{k} \sin (\pi w(k))}{\psi_{f_{1}}^{\prime}\left(t_{k}\right)\left(N+\frac{1}{2}-t_{k}\right)}\right| \\
& \quad \geq C_{1}\left|\sum_{k=-N}^{N} \frac{(-1)^{k} \sin (\pi w(k))}{\psi_{f_{1}}^{\prime}\left(t_{k}\right)\left(N+\frac{1}{2}-t_{k}\right)}\right| .
\end{aligned}
$$

Moreover, since $\psi_{f_{1}}$ is a sine-type function we have either $\psi_{f_{1}}^{\prime}\left(t_{k}\right)=$ $(-1)^{k} c_{k}$ or $\psi_{f_{1}}^{\prime}\left(t_{k}\right)=(-1)^{k+1} c_{k}$ for some positive $c_{k}$, satisfying $\inf _{k \in \mathbb{Z}} c_{k}>0$ and $\bar{c}:=\sup _{k \in \mathbb{Z}} c_{k}<\infty$ [2, p. 164]. We continue the proof, assuming that $\psi_{f_{1}}^{\prime}\left(t_{k}\right)=(-1)^{k} c_{k}, k \in \mathbb{Z}$. The other case is treated analogously. Applying Lemma 3, it follows that

$$
\left|\sum_{k=-N}^{N} \frac{(-1)^{k} \sin (\pi w(k))}{\psi_{f_{1}}^{\prime}\left(t_{k}\right)\left(N+\frac{1}{2}-t_{k}\right)}\right| \geq \frac{1}{\bar{c}} \sum_{k=-N}^{N} \frac{\sin (\pi w(k))}{\left(N+\frac{1}{2}-k\right)}-C_{3}
$$

for all sufficiently large $N \in \mathbb{N}$. Combining all partial results, we have

$$
\left|\sum_{k=-N}^{N} f_{1}\left(t_{k}\right) \frac{\psi_{f_{1}}\left(N+\frac{1}{2}\right)}{\psi_{f_{1}}^{\prime}\left(t_{k}\right)\left(N+\frac{1}{2}-t_{k}\right)}\right| \geq \frac{C_{1}}{\bar{c}} \sum_{k=-N}^{N} \frac{\sin (\pi w(k))}{\left(N+\frac{1}{2}-k\right)}-C_{4}
$$

for all $N \in \mathbb{N}$, sufficiently large. Finally, applying Lemma 4 gives

$$
\begin{aligned}
& \limsup _{N \rightarrow \infty}\left(\sup _{t \in \mathbb{R}}\left|\sum_{k=-N}^{N} f_{1}\left(t_{k}\right) \frac{\psi_{f_{1}}(t)}{\psi_{f_{1}}^{\prime}\left(t_{k}\right)\left(t-t_{k}\right)}\right|\right) \\
& \quad \geq \limsup _{N \rightarrow \infty}\left|\sum_{k=-N}^{N} f_{1}\left(t_{k}\right) \frac{\psi_{f_{1}}\left(N+\frac{1}{2}\right)}{\psi_{f_{1}}^{\prime}\left(t_{k}\right)\left(N+\frac{1}{2}-t_{k}\right)}\right|=\infty,
\end{aligned}
$$

which completes the proof.

\section{CONCLUSION}

In this paper we have studied the adaptive signal reconstruction scheme (4), where the reconstruction functions and the sampling points are matched to the signal. Due to the transformation (3) that is used, this problem is closely related to the reconstruction of bandlimited signals from their sine wave crossings. Since, under the assumptions that $f \in \mathcal{P} \mathcal{W}_{\pi}^{1}$ is real valued on the real axis and $\|f\|_{\infty}<1$, the sampling points are the zeros of a sine-type function, we could employ the theory of sine-type functions to obtain our results. We have shown the uniform convergence of the reconstruction process for the spaces $\mathcal{P} \mathcal{W}_{\pi}^{p}, 1<p \leq \infty$. However, the adaptivity cannot prevent the divergence of the peak value of the approximation process for certain signals in $\mathcal{P} \mathcal{W}_{\pi}^{1}$, which is the largest space in the scale of Paley-Wiener spaces.

\section{REFERENCES}

[1] H. B. Voelcker and A. A. G. Requicha, "Clipping and signal determinism: Two algorithms requiring validation," IEEE Trans. Commun., vol. 21, no. 6, pp. 738-744, June 1973.

[2] B. Ya. Levin, Lectures on Entire Functions, AMS, 1996.

[3] A. A. G. Requicha, "The zeros of entire functions: Theory and engineering applications," Proc. IEEE, vol. 68, no. 3, pp. 308-328, March 1980.

[4] F. E. Bond and C. R. Cahn, "On sampling the zeros of bandwidth limited signals," IRE Trans. Inf. Theory, vol. 4, no. 3, pp. 110-113, September 1958.

[5] E. Masry and S. Cambanis, "Consistent estimation of continuous-time signals from nonlinear transformations of noisy samples," IEEE Trans. Inf. Theory, vol. 27, no. 1, pp. 84-96, January 1981.

[6] I. Bar-David, "An implicit sampling theorem for bounded bandlimited functions," Information and Control, vol. 24, no. 1, pp. 36-44, January 1974.

[7] K. Piwnicki, "Modulation methods related to sine-wave crossings," IEEE Trans. Commun., vol. 31, no. 4, pp. 503-508, April 1983.

[8] R. Duffin and A. C. Schaeffer, "Some properties of functions of exponential type," Bull. Amer. Math. Soc., vol. 44, no. 4, pp. 236-240, April 1938.

[9] H. B. Voelcker, "Toward a unified theory of modulation-part II: Zero manipulation," Proc. IEEE, vol. 54, no. 5, pp. 735755, May 1966.

[10] J. R. Higgins, Sampling Theory in Fourier and Signal Analysis - Foundations, Oxford University Press, 1996.

[11] R. M. Young, An Introduction to Nonharmonic Fourier Series, Academic Press, 2001.

[12] K. Yao and J. B. Thomas, "On some stability and interpolatory properties of nonuniform sampling expansions," IEEE Trans. Circuits Syst., vol. 14, no. 4, pp. 404-408, December 1967.

[13] H. Boche and U. J. Mönich, "Local and global convergence behavior of non-equidistant sampling series," in Proc. IEEE Int. Conf. Acoustics, Speech, and Signal Process., April 2009, pp. 2945-2948.

[14] R. O. Hryniv and Y. V. Mykytyuk, "On zeros of some entire functions," Trans. Amer. Math. Soc., vol. 361, no. 4, pp. 2207 2223, April 2009. 